\title{
Destrucción de aceites dieléctricos mediante oxidación en agua supercrítica: hacia una alternativa de proceso para tratamiento de bifenilos policlorados (PCBs)
}

\author{
Víctor F. Marulanda, Gustavo E. Bolaños ${ }^{\S}$ \\ Escuela de Ingeniería Química, Universidad del Valle, Cali, Colombia \\ §email: gbolano@univalle.edu.co
}

(Recibido: Septiembre 28 de 2009 - Aceptado: Diciembre 01 de 2009)

\begin{abstract}
Resumen
La oxidación en agua supercrítica ha demostrado ser eficaz en la destrucción de un amplio espectro de contaminantes orgánicos persistentes, incluidos los PCBs, de los cuales nuestro país cuenta con amplio inventario. En el presente trabajo se investiga la oxidación en agua supercrítica de aceite dieléctrico usado de transformadores libre de PCBs, como una primera etapa para determinar las condiciones de operación apropiadas que permitan destruir de una forma ambientalmente aceptable los aceites dieléctricos contaminados con PCBs. Todas las combinaciones de tratamientos del diseño factorial propuesto en esta investigación permiten obtener una destrucción prácticamente completa del aceite dieléctrico, las cuales resultan ser condiciones de operación moderadas y alcanzables a nivel industrial. Este trabajo, junto con trabajos similares de destrucción de residuos de PCBs, permite concluir que bajo las condiciones de operación propuestas para destruir el aceite dieléctrico puede ser factible destruir todos los PCBs por debajo del límite de detección siempre y cuando se garantice un tiempo de residencia superior a 15 segundos.
\end{abstract}

Palabras clave: Oxidación en agua supercrítica, Aceites dieléctricos, Bifenilos policlorados, $\mathrm{PCBs}$

CHEMICAL ENGINEERING

\section{Destruction of dielectric oils by supercritical water oxidation: towards an alternative process for the treatment of polyclorinated biphenyls (PCBs)}

\begin{abstract}
Supercritical water oxidation has proved to be effective in the destruction of a wide spectrum of persistent organic pollutants, including PCBs, of which our country has an important inventory. In the present work supercritical water oxidation of waste dielectric oils without PCBs is studied as a first step in determining the appropriate operating conditions that allow dielectric oils contaminated with PCBs to be destroyed, in an environmentally friendly manner. All of the experimental treatments produce practically total destruction of the dielectric oil, at conditions that turn out to be mild and industrially obtainable. This work and several other studies on dielectric oil destruction, indicate that at the established operating conditions it may be feasible to destroy all of the PCBs below the detection limit as long as a residence time longer than 15 seconds is guaranteed.
\end{abstract}

Keywords: Supercritical water oxidation, Dielectric oils, Polyclorinated biphenyls, PCBs 


\section{Introducción}

La oxidación en agua supercrítica o SCWO de las iniciales del nombre en ingles supercritical water oxidation, es un innovador proceso de tratamiento de residuos orgánicos que se conduce a temperaturas y presiones superiores a las del punto crítico del agua $\left(374.2^{\circ} \mathrm{C}\right.$ y 221 bar). En este estado, el agua tiene propiedades fascinantes como medio de reacción. Por ejemplo, la constante dieléctrica es mucho más baja que la del agua a temperatura ambiente $\mathrm{y}$ el número $\mathrm{y}$ persistencia de los puentes de hidrógeno es reducido. Como consecuencia, el agua supercrítica se comporta como un solvente apolar, lo cual le confiere miscibilidad total con cualquier componente orgánico. Los gases como el oxígeno son por su parte completamente solubles en agua supercrítica, por lo que el uso de esta como medio de reacción permite conducir reacciones de oxidación en una fase fluida densa, lo cual, bajo condiciones convencionales de temperatura y presión, ocurriría en un sistema multifásico con limitaciones de transferencia de masa interfacial (Shaw et al., 1991).

Durante los últimos años, la tecnología de oxidación en agua supercrítica ha demostrado ser un proceso eficaz para destruir una gran variedad de residuos industriales y contaminantes orgánicos persistentes que no pueden manejarse adecuadamente por medio de tecnologías convencionales de tratamiento de residuos. Se han reportado eficiencias de destrucción cercanas al $100 \%$ para compuestos nitrogenados como la piridina, la anilina, el nitrobenceno y el amoniaco (Cocero et al., 2000, Crain et al., 1993, Zhang y Hua, 2003), así como para compuestos fenólicos (Perez et al., 2004, Son et al., 2008,) y residuos menos convencionales como agentes de armas químicas obsoletas, desechos radiactivos, y aguas residuales industriales de una amplia variedad de procesos químicos (Kritzer y Dinjus, 2001, Goto et al., 1998, Park et al., 2003). Sin embargo, la mayor atención se ha centrado en la aplicación de la oxidación en agua supercrítica para la destrucción de bifenilos policlorados o PCBs (Hatakeda et al., 1999, Anitescu et al., 2004, Anitescu y Tavlarides, 2000).

La Figura 1 muestra la formula estructural de los

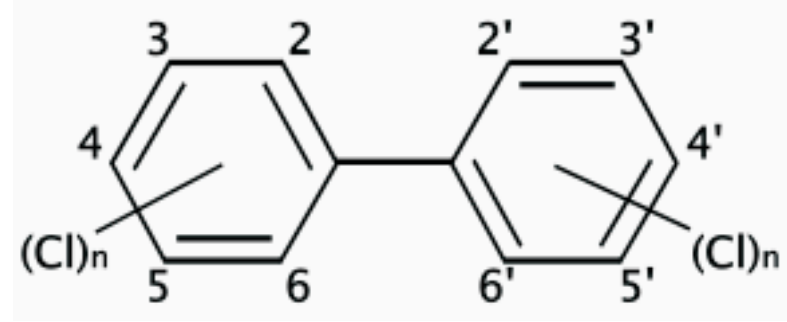

Figura 1. Fórmula estructural de los PCBs

PCBs. Los PCBs son compuestos orgánicos aromáticos que fueron creados por el hombre y que se componen de dos anillos de fenilos con átomos de cloro. De acuerdo a la cantidad y posición de los átomos de cloro en ambos anillos se conforman diferentes congéneres de PCB. Existen 209 posibles cóngeneres, de los cuales, cerca de 130 se utilizan en productos comerciales. Los PCBs son compuestos muy estables, resistentes a la degradación térmica, química y biológica, altamente tóxicos y posiblemente cancerigenos y mutagénicos (Fang et al., 2005). Se acumulan en el tejido adiposo y se biomagnifican en las criaturas del extremo superior de la cadena alimenticia (PNUMA, 2003). Son líquidos, viscosos, incoloros o de color amarillo pálido, con leve olor a hidrocarburos. Poseen excelentes propiedades aislantes, longevidad y no son inflamables, por lo cual se utilizaron ampliamente en equipos eléctricos como transformadores y condensadores, en intercambiadores de calor, sistemas hidráulicos y también en la fabricación de pinturas y plásticos. Se fabricaron entre 1930 y 1980, año en que su producción fue prohibida.

El Convenio de Estocolmo sobre contaminantes orgánicos persistentes, ratificado por Colombia en 2001, requiere que los países firmantes adopten medidas para reducir el uso y eliminar de una manera ambientalmente racional los equipos, los líquidos y los desechos contaminados con PCBs tan pronto como sea posible, pero a más tardar antes de 2028. El inventario preliminar de PCBs en Colombia, basado en la información disponible sobre existencias confirmadas de PCBs y estudios de campo realizados en aceites y equipos eléctricos, da cuenta de la existencia de 10073 a 
13199 toneladas de residuos de PCBs, de los cuales solo alrededor de 1200 toneladas corresponden a PCBs puros en tranformadores en uso y desuso. El restante corresponde a aceites dieléctricos contaminados con PCBs en un amplio rango de concentraciones (Ministerio de Medio Ambiente Vivienda y Desarrollo Territorial Republica de Colombia, 2007). Esta contaminación es resultado de una práctica muy común hasta hace algunos años en el sector eléctrico, la cual consistía en rellenar con aceite mineral nuevo, transformadores que previamente contenían aceite PCB puro.

Entre las alternativas tecnológicas existentes para la eliminación de los desechos de PCBs se cuentan la incineración a temperaturas alrededor de 1200 ${ }^{\circ} \mathrm{C}$, la reducción química, la deshalogenación, la bioremediación, la pirolisis, el arco de plasma y más recientemente la oxidación en agua supercrítica (Tavlarides et al., 2000). Muchas de estas tecnologías no han pasado de escala de laboratorio debido a que los resultados de la destrucción no han sido del todo prometedores. La incineración es la única tecnología de tratamiento que se acepta internacionalmente para la eliminación de PCBs (Rahuman et al., 2000). La incineración de PCBs comúnmente genera los contaminantes orgánicos persistentes más peligrosos conocidos por el hombre, como son los dibenzofuranos y las dioxinas policloradas (PCDFs/PCDDs) (Anitescu et al., 2000). Estos componentes indeseados junto con los precursores de la lluvia acida, $\mathrm{NO}_{\mathrm{x}} \mathrm{y} \mathrm{SO}_{\mathrm{x}}$, se producen en la combustión de PCBs, pero no se forman durante las reacciones a más baja temperatura que ocurren en la oxidación supercrítica (Anitescu et al., 2000). Adicionalmente, en Colombia no hay instalaciones de incineración apropiadas para tal fin por lo que la disposición debe realizarse en países como Francia o Canadá, con unos costos que resultan prohibitivos para cualquier industria.

En este trabajo se estudia la oxidación en agua supercrítica de aceites dieléctricos usados, libres de PCBs, como una primera aproximación al desarrollo de un proceso para tratamiento de aceites contaminados en el rango de concentraciones de 50 a $1000 \mathrm{mg} / \mathrm{L}$. La validez de esta aproximación se fundamenta en estudios de tratamiento similares (Kim, 2006) donde se ha logrado la completa destrucción de los PCBs de aceite dieléctrico contaminado a condiciones de operación bajo las cuales no ha sido posible destruir completamente la carga orgánica del aceite. Con el fin de determinar las condiciones óptimas de operación del proceso para lograr una destrucción total del aceite dieléctrico, se evaluó el efecto de la temperatura, el porcentaje de exceso de agente oxidante y la concentración del aceite dieléctrico sobre la destrucción de la materia orgánica expresada como carbono orgánico total (COT). Los resultados experimentales permiten concluir que mediante este proceso es posible obtener eficiencias de destrucción del contenido orgánico del aceite cercanas al $100 \%$.

\section{Metodología}

Las corridas de oxidación en agua supercrítica se llevaron a cabo en una unidad continua a escala banco diseñada y construida en el Laboratorio de Fluidos Supercríticos de la Universidad del Valle, similar en esencia, mas no en detalle, a las reportadas por varios investigadores (Zhang et al., 2003, Pérez et al., 2004). El equipo permite operar a temperaturas y presiones hasta de $600{ }^{\circ} \mathrm{C}$ y $34 \mathrm{MPa}$ respectivamente y una velocidad de flujo máxima de $46 \mathrm{~mL} / \mathrm{min}$. La Figura 2 muestra el esquema tecnológico de la unidad continua construida, en la cual se distinguen cuatro zonas: (1) la zona de presurización, compuesta por bombas neumáticas de alta presión marca Williams, modelo CP250V225, caudal máximo de $41.6 \mathrm{~mL} / \mathrm{min}$ y presión máxima de descarga de $49.6 \mathrm{MPa}$ para el agente oxidante y CP125V125, caudal máximo de $4.6 \mathrm{~mL} / \mathrm{min}$ y presión máxima de descarga de 59.6 MPa para el contaminante orgánico a oxidar, con una presión de suministro de aire comprimido de $0.75 \mathrm{MPa}$, y tanques de almacenamiento de acero inoxidable para el agente oxidante y el componente orgánico a tratar. El agente oxidante es peróxido de hidrógeno al $8 \%$ en peso. Un disco de ruptura de $41 \mathrm{MPa}$ ubicado a la salida de la bomba de presurización del peróxido protege al sistema de sobrepresurizaciones. (2) la zona de reacción y precalentamiento, compuesta por un baño isotérmico de arena fluidizada con resistencias eléctricas de $2000 \mathrm{~W}$. Las corrientes procedentes de las bombas de alta presión se precalientan a la temperatura de reacción en sendos serpentines construidos con tubería de alta presión marca 


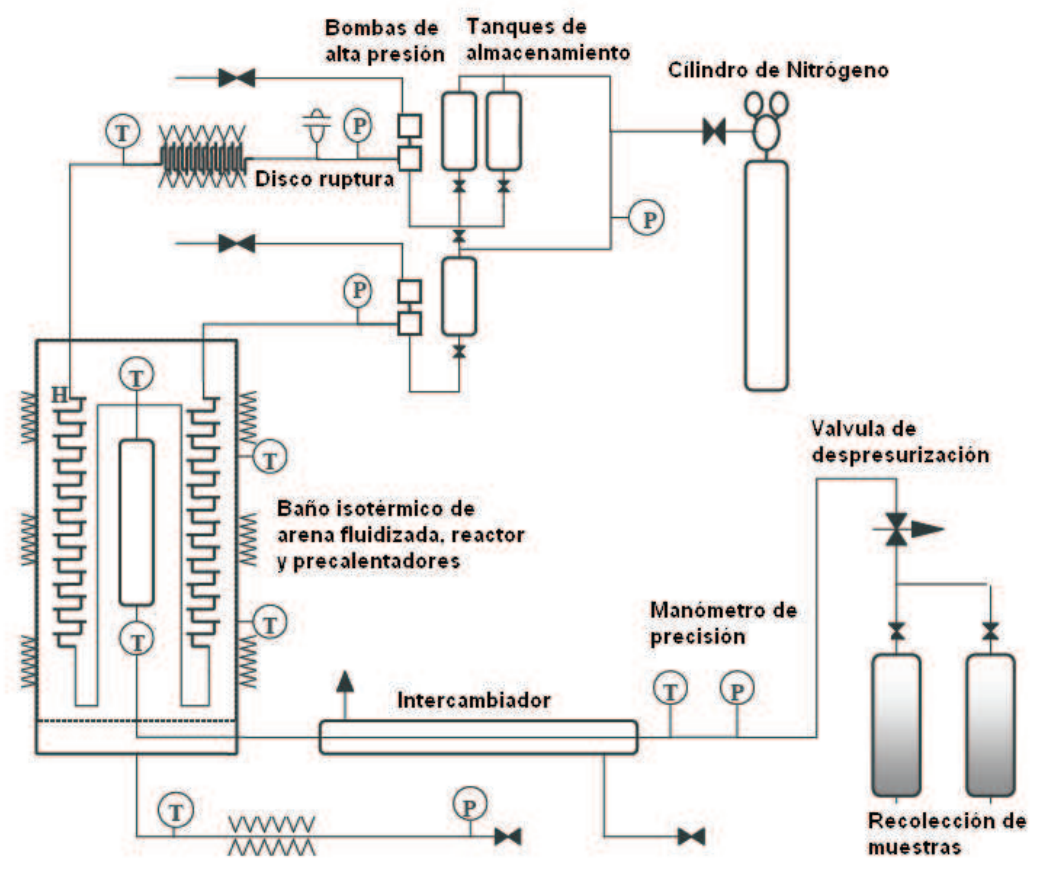

Figura 2. Esquema tecnológico de la unidad continua construida

Swagelok de $5 \mathrm{~m}$ de largo, $0.065 \mathrm{~cm}$ O.D. y 0.038 cm I.D. y sumergidos en el baño de arena. Los serpentines se encuentran en una tee de alta presión que sirve a la vez como mezclador de choque antes de entrar al reactor tubular, el cual está construido con tubería de alta presión de 1.27 cm O.D. y $66 \mathrm{~cm}$ de largo. Las temperaturas a la entrada y salida del reactor y en el cuerpo del baño isotérmico se miden por medio de termocuplas tipo $\mathrm{K}$ fabricadas en Inconel para resistir las severas condiciones de operación del sistema. La temperatura del baño se regula por medio de un controlador PID marca Desin Instruments modelo BS-1400 con una desviación máxima de 2 grados en el set point. (3) La zona de enfriamiento y despresurización, compuesta por un intercambiador de calor de tubos concéntricos con agua como medio de refrigeración, un manómetro Ashcroft de precisión modelo A4A y certificado de trazabilidad NIST y 12" de carátula y una válvula de aguja con sellos de grafito marca Autoclave Engineers modelo 20SM4081-6Y la cual regula la presión de todo el sistema con una desviación máxima de $0.3 \mathrm{MPa}$ y el caudal de salida del reactor y (4) la zona de separación de fases y recolección de productos, la cual consta de un recipiente de vidrio con tubuladura lateral para la evacuación de la fase gaseosa hacia un sistema de extracción y lavado de gases, y un puerto de muestreo en la parte inferior para permitir la recolección de las muestras para análisis.

En una corrida de oxidación en agua supercrítica típica se cargan la solución de peróxido y el aceite dieléctrico en los tanques de alimentación. Debido a la elevada viscosidad, lo cual dificulta el bombeo, y alta carga orgánica del aceite dieléctrico, se formó una emulsión de $6 \mathrm{~mL}$ del aceite en un litro de agua deionizada y destilada por medio del emulsificante comercial Arkopal ${ }^{\circledR}$ $\mathrm{y}$ un proceso de agitación por medio de una sonda ultrasónica. Este proceso permitió utilizar una mínima cantidad de emulsionante y obtener una emulsión que se mantuvo estable durante varios meses, de viscosidad similar a la del agua. Trabajos similares han reducido la viscosidad del aceite a oxidar por medio de solventes orgánicos como el metanol, el benceno y el hexano (Anitescu et al., 2005). El uso de solventes tiene la desventaja de requerir una mayor cantidad de oxígeno para destruir de forma simultánea el contaminante y el solvente, lo cual incrementa los 
costos del proceso tanto por el solvente orgánico como por el oxidante.

El caudal de las bombas de alta presión se fija atendiendo los requerimientos de oxígeno necesario para lograr una destrucción completa de la carga orgánica de la emulsión y del tiempo de residencia deseado para el volumen de reacción disponible, a la temperatura y presión de operación seleccionadas. Anitescu y colaboradores (Anitescu et al., 2004) proporcionan una explicación detallada de la forma como se calcula el tiempo de residencia a las condiciones de operación atendiendo estos requerimientos. Una vez iniciado el bombeo a la temperatura de operación, se regula la presión del sistema por medio de la válvula de aguja. Durante el arranque del equipo y cada vez que una de las condiciones de reacción cambia, existe un período de transitoriedad en el sistema, caracterizado por la inestabilidad de las temperaturas de entrada y salida del reactor y del caudal de salida. Se ha establecido en el procedimiento experimental del equipo la recolección de $100 \mathrm{~mL}$ de muestra en estado transitorio antes de recolectar la muestra para análisis. Este procedimiento ha demostrado ser satisfactorio. Las muestras tomadas se mantuvieron a $4{ }^{\circ} \mathrm{C}$ en frascos de vidrio ambar sin dejar espacio vacío para evitar la pérdida de volátiles. Las muestras se analizaron por medio de un equipo de carbono orgánico total marca Shimadzu, modelo TOC-VCPH con un límite de detección de $0.112 \mathrm{mg} / \mathrm{L}$.
Para la exploración de las condiciones óptimas de operación se corrió un diseño experimental $2^{3}$ y dos puntos centrales con los factores temperatura (A), porcentaje de exceso de agente oxidante sobre el teórico requerido para conversión completa de la materia orgánica (B) y caudal de la emulsión del componente orgánico en $\mathrm{mL} / \mathrm{min}$ (C), tal como se muestra en la Figura 3. La selección de los niveles bajo y alto de la temperatura, $520^{\circ} \mathrm{C}$ y $550^{\circ} \mathrm{C}$, y del agente oxidante, $300 \%$ y $400 \%$, se hizo en base a los resultados experimentales de destrucción de aceite dieléctrico contaminado con PCBs reportados en la literatura (Kim, 2006), mientras que la elección de los niveles alto y bajo del caudal de componente orgánico, $1.15 \mathrm{~mL} / \mathrm{min}$ y 2.3 $\mathrm{mL} / \mathrm{min}$, se hizo con el propósito de mantener un tiempo de residencia superior a 15 segundos en todas la corridas del diseño experimental. La presión de operación se mantuvo constante en 3600 psi (24.1 MPa) para todas las corridas del diseño experimental. La selección de esta presión se basó en los resultados experimentales de corridas exploratorias del efecto de la presión a temperatura constante en la eficiencia de la destrucción de la materia orgánica, representada como remoción de la coloración, donde se verificó que el incremento de esta produce una disminución del porcentaje de destrucción de la materia orgánica. La carga orgánica de la emulsión de aceite dieléctrico se determinó por medio del análisis de carbono orgánico total, obteniéndose un valor de $11640 \mathrm{mg} / \mathrm{L}$. Debido a la

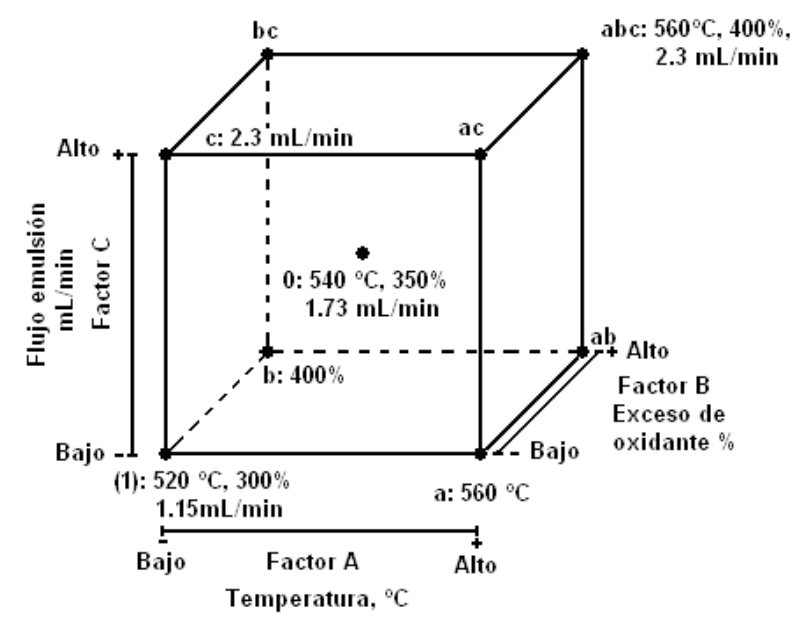

Figura 3. Diseño experimental de tres factores a dos níveles aumentado con dos puntos centrales 
compleja composición del aceite dieléctrico, el cual está formado por un amplio rango de componentes cíclicos similares al ciclohexano, no fue posible saber de antemano la carga orgánica de la emulsión, por lo que se supuso que el aceite estaba constituido únicamente por ciclohexano. Con esta suposición se esperaba un valor de COT de alrededor de $4000 \mathrm{mg} / \mathrm{L}$. Obviamente este parámetro fue subestimado durante el planteamiento del diseño experimental.

\section{Resultados y discusión}

La Tabla 1 muestra los resultados experimentales del proceso de oxidación en agua supercrítica de aceite dieléctrico usado libre de PCBs. La Temperatura es el promedio de las temperaturas de entrada y salida del reactor. El flujo de oxidante en $\mathrm{mL} / \mathrm{min}$ a las condiciones de salida de la bomba se determinó por medio del flujo alimentado de la emulsión y el exceso de oxígeno especificado para esa corrida en el diseño factorial. El flujo total a las condiciones de temperatura y presión a la entrada del reactor se calculó por medio de un balance de masa entre la salida de las bombas a $25^{\circ} \mathrm{C}$ y 3600 psi y la entrada del reactor a la temperatura de reacción y 3600 psi (Anitescu et al., 2004). La densidad de la mezcla se tomó como la densidad del agua ya que esta constituye más del $95 \%$ en peso de la mezcla reaccionante. Con el flujo a las condiciones de reacción se calculó el tiempo de residencia de acuerdo a la Ec (1):

$$
\tau=\frac{V}{v}
$$

Donde $\mathrm{V}$ es el volumen del reactor en $\mathrm{mL}$ y $v$ el flujo volumétrico a la entrada del reactor. La carga orgánica se determinó por medio de la técnica instrumental de carbono orgánico total (COT). La conversión se define en función de la desaparición del carbono orgánico total de acuerdo a la Ec (2):

$$
X=\frac{C O T_{o}-C O T}{C O T_{o}}
$$

Donde COT。 y COT son el carbono orgánico total a la entrada y sálida del reactor respectivamente. La determinación del avance de la reacción en función del carbón orgánico total, en especial cuando se tienen mezclas complejas de diversos componentes, se fundamenta en el hecho de que las reacciones de oxidación proceden a través de una serie de intermediarios, algunos muy refractarios como el ácido acético, que se constituyen al mismo tiempo en el paso limitante de la velocidad de oxidación a dióxido de carbono y nitrógeno (Mateos, et al., 2005).

En la Tabla 1 se puede observar que todas las combinaciones de tratamientos del diseño experimental produjeron conversiones de la materia orgánica superiores al 99\%, por lo que el análisis de varianza de los mismos no arrojaría efectos estadísticamente significativos de alguno de los factores principales o interacciones de orden superior. Sin embargo, es posible efectuar una interpretación de los resultados experimentales por medio de las gráficas de los efectos de los factores y sus interacciones. La Figura 4 muestra el efecto de los factores temperatura (A), exceso de agente oxidante (B), flujo volumétrico de la emulsión (C) y la interacción $\mathrm{AB}$ en la conversión del proceso de oxidación en agua supercrítica de aceite dieléctrico usado libre de PCBs.

En la Figura 4 se observa que al analizar los factores A, B y C separadamente se obtendrían las conversiones más altas en el nivel bajo de la temperatura y caudal de orgánico y el nivel alto del exceso de oxígeno. A una conclusión diferente se llega al analizar la gráfica de la interacción $\mathrm{AB}$.

La mayor conversión se obtendría al utilizar en el nivel bajo de A, tal como se concluyó de las gráficas de los efectos de los factores individuales, y el nivel bajo de $\mathrm{B}$, ya que este produciría el mismo resultado que el nivel alto con un consumo de oxígeno menor.

Estos resultados pueden explicarse al considerar el tiempo de residencia de las corridas, el cual depende fuertemente de la temperatura y el caudal alimentado al reactor. Teóricamente, una mayor temperatura producirá una mayor velocidad de reacción y por ende una mayor conversión. Sin embargo, al aumentar la temperatura a presión constante disminuye la densidad de la fase supercrítica, es decir que la masa de fluido ocupa un volumen mayor cuando incrementa la temperatura debido a la expansión térmica, por lo 
cual tendrá un tiempo de residencia menor en el reactor. El efecto del caudal en el tiempo de residencia es evidente, pues al disminuir el caudal de orgánico se requerirá un caudal de oxígeno menor y por tanto un caudal menor alimentado al reactor, con lo cual se obtienen un tiempo de residencia mayor.
Estos resultados demuestran que es posible operar el proceso de oxidación en agua supercrítica y lograr una muy elevada destrucción de la materia orgánica sin la formación de subproductos indeseados, a temperaturas y porcentajes de exceso de oxígeno moderados, $520{ }^{\circ} \mathrm{C}$ y $300 \%$ respectivamente, condiciones que son factibles de alcanzar en una planta piloto. Otros estudios de oxidación en agua supercrítica han empleado

Tabla 1. Resultados experimentales del proceso de oxidación en agua supercrítica de aceite dieléctrico usado

\begin{tabular}{|c|c|c|c|c|c|c|c|}
\hline \multirow{2}{*}{$\begin{array}{c}\text { Temperatura } \\
\left({ }^{\circ} \mathrm{C}\right)\end{array}$} & \multirow{2}{*}{$\begin{array}{c}\text { Exceso de } \\
\text { oxidante (\%) }\end{array}$} & \multicolumn{2}{|c|}{ Flujo (mL/min) } & \multirow{2}{*}{$\begin{array}{l}\text { Tiempo de } \\
\text { residencia (s) }\end{array}$} & \multicolumn{2}{|c|}{ Carbono orgánico total $(\mathrm{mg} / \mathrm{L})$} & \multirow[t]{2}{*}{ Conversiór } \\
\hline & & Orgánico & Oxidante & & Inicial & Final & \\
\hline 520.0 & 300 & 1.150 & 6 & 43.38 & 1872.0 & 3.41 & 0.998 \\
\hline 552.8 & 300 & 1.150 & 6 & 40.00 & 1872.0 & 1.09 & 0.999 \\
\hline 524.5 & 300 & 2.300 & 12 & 21.70 & 1872.0 & 3.60 & 0.998 \\
\hline 522.0 & 400 & 2.300 & 15 & 17.93 & 1547.5 & 3.30 & 0.998 \\
\hline 538.6 & 350 & 1.725 & 10 & 25.30 & 1712.5 & 1.80 & 0.999 \\
\hline 557.5 & 400 & 2.300 & 15 & 16.50 & 1547.5 & 6.60 & 0.996 \\
\hline 551.3 & 400 & 1.150 & 7.5 & 33.00 & 1547.5 & 2.26 & 0.998 \\
\hline 556.8 & 300 & 2.300 & 12 & 20.00 & 1872.0 & 7.80 & 0.995 \\
\hline 519.0 & 400 & 1.150 & 7.5 & 35.86 & 1547.5 & 1.74 & 0.998 \\
\hline 535.8 & 350 & 1.725 & 10 & 25.30 & 1712.5 & 2.79 & 0.998 \\
\hline
\end{tabular}

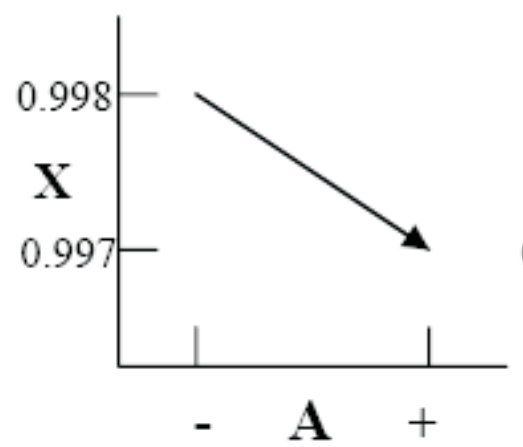

- A +
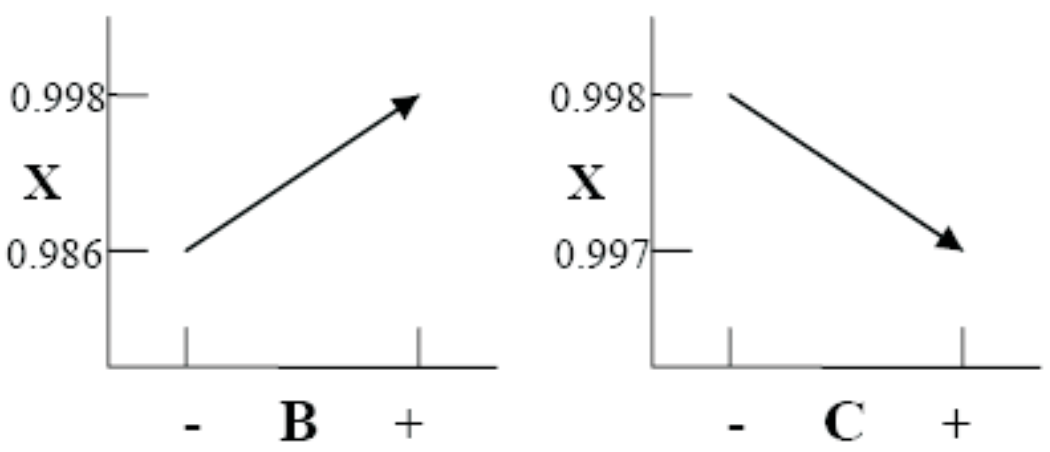

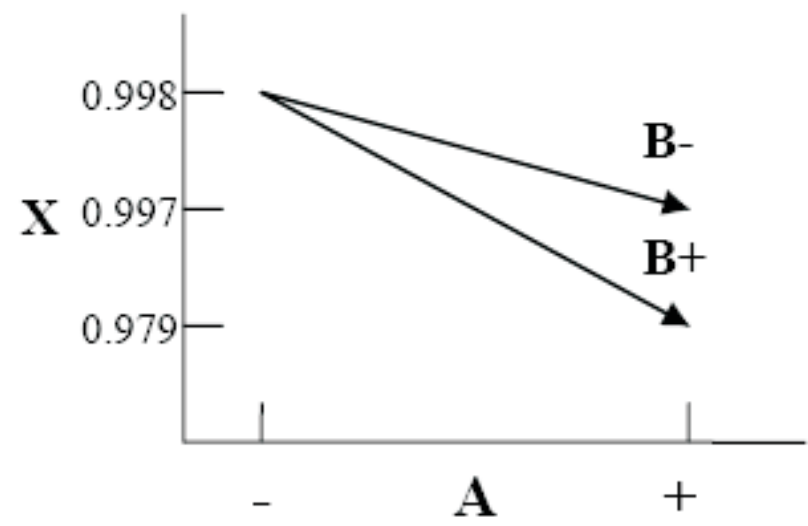

Figura 4: Efecto de los factores temperatura (A), exceso de agente oxidante (B), flujo volumétrico de la emulsión, (C) y la interacción $A B$ 
temperaturas de reacción de alrededor de $700{ }^{\circ} \mathrm{C}$ (Cocero et al., 2000) y porcentajes de exceso de oxígeno tan altos como 4000\% (Aki y Abraham, 1999).

Kim (2006) reportó destrucción de PCBs en aceites dieléctricos a niveles por debajo del límite de detección $(<0.1 \mathrm{mg} / \mathrm{L})$. En su trabajo utilizó una emulsión de aceite dieléctrico contaminado con $1 \mathrm{mg} / \mathrm{L}$ de PCBs y un contenido inicial de carbono orgánico total de $10000 \mathrm{mg} / \mathrm{L}$ a $500{ }^{\circ} \mathrm{C}$, porcentajes de exceso de agente oxidante de $400 \%$ $\mathrm{y}$ tiempos de residencia superiores a 15 segundos. En este trabajo se produjeron cantidades identificables de PCBs solo en las corridas con tiempos de residencia menores a 5 segundos. A pesar de la casi destrucción total de los PCBs por medio de estas condiciones de operación, no se obtuvo una conversión completa $(>99.9 \%)$ del carbono orgánico total del producto. Como se observa en la Tabla 1, utilizando una carga orgánica similar a la empleada en el estudio de Kim (2006) para las corridas experimentales, se obtuvo una destrucción de la materia orgánica hasta un valor mínimo de $1 \mathrm{mg} / \mathrm{L}$, lo cual puede atribuirse al mayor tiempo de residencia utilizado en este estudio, el cual es de alrededor de 30 segundos, en comparación con el de Kim (2006) que fue solo de 15 segundos y obtuvo una destrucción de la materia orgánica hasta un valor mínimo de $100 \mathrm{mg} / \mathrm{L}$.

\section{Conclusiones}

Los resultados experimentales de la oxidación en agua supercrítica de aceites dieléctricos usados libres de PCBs y la comparación con resultados previamente publicados para aceites similares, permiten concluir que bajo las condiciones de operación del proceso propuestas puede ser posible efectivamente destruir los PCBs y la totalidad de la carga orgánica del aceite dieléctrico en un proceso continuo, sin generación de subproductos indeseados que suelen presentarse en los procesos de destrucción a temperaturas mayores, como la incineración. Las condiciones de reacción bajo las cuales se obtuvo la mayor conversión $510{ }^{\circ} \mathrm{C}$ y $350 \%$ de exceso de agente oxidante, son condiciones de operación que resultan moderadas en comparación con las empleadas en otros estudios de oxidación en agua supercrítica, y hacen factible la implementación de este proceso a nivel industrial para dar solución a la problemática originada por desechos de difícil disposición y manejo, entre ellos los PCBs.

\section{Agradecimientos}

Los autores desean expresar su agradecimiento a COLCIENCIAS por el apoyo financiero del proyecto de investigación No. 1106-08-17745, contrato No.364-2005.

\section{Referencias Bibliográficas}

Aki, S., \& Abraham, M. A. (1999). Catalytic supercritical water oxidation of pyridine: comparison of catalysts. Industrial \& Engineering Chemistry Research. 38 (2), 358-367.

Anitescu, G., \& Tavlarides, L. L. (2000). Oxidation of aroclor 1248 in supercritical water: a global kinetic study. Industrial \& Engineering Chemistry Research 39(3), 583-591.

Anitescu, G., Tavlarides, L. L., \& Munteanu, V. (2004). Decomposition of monochlorobiphenyl isomers in supercritical water in the presence of methanol. AIChE Journal 50 (7), 1536-1544.

Anitescu, G., Munteanu, V., \& Tavlarides, L. L. (2005). Co-oxidation effects of methanol and benzene on the decomposition of 4chlorobiphenyl in supercritical water. The Journal of Supercritical Fluids 33 (2), 139-147.

Cocero, M. J., Alonso, E., Torío, R., Vallelado, D., \& Fdz-Polanco, F. (2000). Supercritical water oxidation in a pilot plant of nitrogenous compounds: 2-propanol mixtures in the temperature range $500-750{ }^{\circ} \mathrm{C}$. Industrial \& Engineering Chemistry Research 39 (10), 37073716 .

Crain, N., Tebbal, S., Li, L., \& Gloyna, E. (1993). Kinetics and reaction pathways of pyridine oxidation in supercritical water. Industrial \& Engineering Chemistry Research 32(10), 22592268. 
Fang, Z., Xu, S. K., Smith, R. L., Arai, K., \& Kozinski, J. A. (2005). Destruction of decachlorobiphenyl in supercritical water under oxidizing conditions with and without $\mathrm{Na}_{2} \mathrm{CO}_{3}$. The Journal of Supercritical Fluids 33 (3), 247-258.

Goto, M., Nada, T., Ogata, A., Kodama, A., \& Hirose, T. (1998). Supercritical water oxidation for the destruction of municipal excess sludge and alcohol distillery wastewater and molasses. The Journal of Supercritical Fluids 13 (1), 277-282.

Hatakeda, K., Ikushima, Y., Sato, O., Aizawa, T., \& Saito, N. (1999). Supercritical water oxidation of polyclorinated biphenyls using hydrogen peroxide. Chemical Engineering Science 54 (15), 3079-3084.

Kim, Y. C. (2006). Supercrittical water oxidation of PCBs-contaminated transformer oil. In Proceedings of the $8^{\text {th }}$ Conference on Supercritical Fluids and Their Applications, Ischia, Italy.

Kritzer, P., \& Dinjus, E. (2001). An assessment of supercritical water oxidation (SCWO): existing problems, possible solutions and new reactor concepts. Chemical Engineering Journal. 83 (3), 207-214.

Mateos, D., Portela, J. R., Mercadier, J., Marias, F., Marraud, C., \& Cansell, F. (2005). New Approach for Kinetic Parameters Determination for Hidrotermal Oxidation Reaction. The Journal of Supercritical Fluids 34 (1), 63-70.

Ministerio de Medio Ambiente, Vivienda y Desarrollo Territorial Republica de Colombia. (2007). Inventario preliminar de compuestos bifenilos policlorados (PCB) existentes en Colombia, ISBN 978-958-97978-4-6.

Park, T. J., Lim, J. S., Lee, Y. W., \& Kim, S. H. (2003). Catalytic supercritical water oxidation of wastewater from terephthalic acid manufacturing process. The Journal of Supercritical Fluids 26 (3), 201-213.
Pérez, I. V., Rogak, S., \& Branion, R. (2004). Supercritical water oxidation of phenol and 2,4dinitrophenol. The Journal of Supercritical Fluids 30 (1), $71-87$.

PNUMA (Programa de las Naciones Unidas para el Medio Ambiente) (2003). Guía del Convenio de Estocolmo sobre Contaminantes Orgánicos Persistentes. Preparado por UNEP Chemicals. Geneve.

Rahuman, M., Pistone, L., Trifiro, F., \& Miertus S. (2000). Destruction technologies for polychlorinated biphenyls. In Proceedings on the Expert Group Meetings on POPs and Pesticides Contamination. Trieste.

Shaw, R. W., Brill, T. B., Clifford, A. A., Eckerd, C. A., \& Franck, E. U. (1991). Supercitical water: a medium for chemistry. Chemical. Engineering News 69 (51), 26-39.

Son, S. Lee, J., Byeon, S., \& Lee, C. (2008). Surface chemical analysis of corroded alloys in subcritical and supercritical water oxidation of 2-chlorophenol in continuous anticorrosive reactor system. Industrial \& Engineering Chemistry Research 47 (7), 2265-2272.

Tavlarides, L. L., Zhou, W., \& Anitescu, G. (2000). Supercritical fluid technology for the remediation of $P C B / P A H$-contaminated soils/sediments. In Proceedings of the 2000 Conferencie on Hazardous Waste Research. Denver, Colorado.

Zhang, G., \& Hua, I. (2003). Supercritical water oxidation of nitrobenzene. Industrial \& Engineering Chemistry Research 42 (2), 285-289. 